\title{
Trajectory Planning and Control of Mobile Manipulator
}

\author{
Bowen Luo ${ }^{1, a^{*}}$, Ying Sun ${ }^{1,2, b}$ and Mingchao $\mathrm{Yu}^{2, \mathrm{c}}$
}

1 Key Laboratory of Metallurgical Equipment and Control Technology, Ministry of Education, Wuhan University of Science and Technology, Wuhan 430081, China.

\section{Hubei Key Laboratory of Mechanical Transmission and Manufacturing Engineering, Wuhan University of Science and Technology, Wuhan 430081, China.}

a378401869@qq.com, bsunying65@wust.edu.cn, c503616860@qq.com.

Keywords: Manipulator; Modularization;Motion planning;Multi mobile manipulator

\begin{abstract}
A manipulator is an automatic operating device that can imitate certain movements of the hand and arm , and it can be used to grab and carry objects or operate tools in a fixed procedure. This paper mainly discusses the concept, the manipulator development history and research trends of the manipulator at home and abroad, it mainly from three aspects of the manipulator motion planning, coordination and control of multiple mobile robots reviews the development in recent years, and introduces the related problems and the corresponding treatment methods. Finally, it explores the development direction and the problem that needs to be solved in this research field.
\end{abstract}

\section{Introduction}

Manipulator is the first appearance of the industrial robot is the first modern robot, it can replace the heavy labor to achieve the production of mechanization and automation, but also in a harmful environment to protect the safety of personal safety, which is widely used in machinery manufacturing, metallurgy, Electronics, light industry and atomic energy and other departments $[1,2]$. With the rapid development of China's economy, the country's growing demand for industrial growth is also growing, the manipulator as a very important tool for industrial production, the continuous breakthrough of its technology has become increasingly important. At present, the robot according to the drive classification can be divided into: hydraulic, pneumatic, electric, mechanical manipulator; according to the scope of application can be divided into two kinds of special robots and general manipulator; according to the movement trajectory control can be divided into point control and continuous trajectory control manipulator. The mobile manipulator is composed of one or a plurality of manipulator and a movable platform, the structure of the manipulator is mounted on the mobile platform of the manipulator has almost infinite work space and more redundancy $[3,4]$. At the same time, the manipulator also has the function of movement and operation, and these advantages make it superior to the mobile robot and the traditional manipulator. The problem of control of mobile manipulator is the hotspot and difficulty of control research in recent years. Our research work in this area has just started,

\section{Development of Manipulators at Home and Abroad.}

In 1958 the United States joint control company developed the first Manipulator. China's first Manipulator in Shanghai, the success of development, followed by the provinces began to develop and apply the Manipulator [5,6]. From the seventh five-year plan (1986 to 1990), our government has greatly increased the importance of industrial Manipulators, and invested a lot of money for this project, with the participation of many scholars and researchers, research and development and Manufacturing a series of industrial Manipulators, including by the Beijing Institute of Mechanical Automation designed and manufactured spray Manipulator, the Guangzhou Machine Tool Research Institute and the Beijing Institute of Machine Tool design and manufacture of spot welding Manipulator, Dalian Machine Tool Institute designed and manufactured by the argon arc welding Manipulator, Shenyang University of Technology design and manufacture of loading and unloading Manipulator and so on. It is worth noting that these Manipulator controllers are 
developed by the Shenyang Institute of Automation, Chinese Academy of Sciences and the Institute of Manipulator, Beijing University of Science and Technology [7]. At the same time, a series of key components of th Manipulator have also been developed, such as Manipulator bearings, shock absorbers, DC servo motors, encoders and so on

\section{Definition of Mobile Manipulators.}

The mobile manipulator is composed of one or a plurality of manipulator and a movable platform, the structure of the manipulator is mounted on the mobile platform of the manipulator has almost infinite work space and more redundancy [8,9]. At the same time, the manipulator also has the function of movement and operation, and these advantages make it superior to the mobile robot and the traditional manipulator. Therefore, it is of great theoretical and practical significance to study the control problem of such systems [10]. There are two main categories of research on the control of mobile manipulator: one is the motion planning problem of mobile platform and manipulator; the other is the coordinated control problem of mobile platform and manipulator. For a given task, it is important to determine the movement of the robot and the platform, When the planning is completed, appropriate algorithms are used for control, Because the mobile Manipulator involves the platform and the Manipulator two subsystems, how to achieve its coordination control is very important. In addition, multiple mobile robots have many practical applications in conjunction with task completion [11].

\section{Mobile Manipulator Movement Planning.}

The so-called planning is in the environment with obstacles in accordance with certain evaluation criteria, looking for a starting from the state to the target state without touch path. For mobile Manipulators, planning issues involve Manipulator planning and platform planning in two parts $[12,13]$. The combination of the platform and the Manipulator makes the system redundant, the same task can be achieved either by a single motion Manipulator or platform, or by moving a Manipulator and a platform at the same time. In addition, the mobile Manipulator system has complex dynamic model, strong dynamic coupling and non-complete constraints that mobile platform may introduce, which makes the planning problem of mobile manipulator, and this paper introduces the existing planning method [14,15]. Early mobile Manipulator planning methods did not fully exploit the capabilities of mobile Manipulators or did not take into account the complete dynamics of mobile Manipulators. Some are limited to the realization of mobile or operation of a function, while others ignore the dynamic characteristics. Yamamoto proposed to maintain the maximum operability of the manipulator area for the preferred operating area (Preferred Operation Region), through the planning of the movement of the platform to make the Manipulator in the preferred operating area; Seraji mobile platform to introduce the degree of freedom and multi-joint introduction of the same degree of freedom, The whole system is regarded as a redundant manipulator, and its online planning is carried out [16].

\section{The Degree of Freedom of Manipulator's Obstacle Avoidance and Its Driving Model.}

According to the number of joint movement axis, can be divided into three kinds: single-axis joint, is in a plane, around a shaft movement of the joint; biaxial joint, can be around the two axes of movement of the joints, in addition to the horizontal axis For the flexion and extension movement, but also along the vertical axis of the left and right swing; multi-axis joints, there are three vertical axis of movement, the joints are mostly spherical or nest, can do a variety of directions of movement, Adduction and outreach, but also do rotary movement [17]. According to the characteristics of the movement of the manipulator, the degree of freedom is configured as follows: the wrist uses a multi-axis joint arm a pitch degree of freedom, arm a pitch degree of freedom, the base of a degree of freedom of rotation, as shown in Figure 1. 


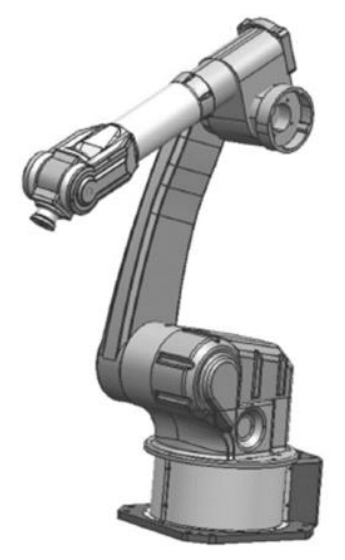

Figure 1. 6 DOF manipulator model

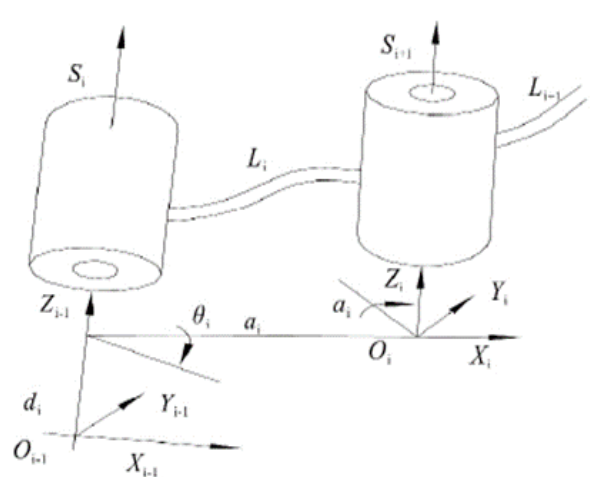

Figure 2. D-H parameter definition

The manipulator is connected by multiple joints, and the joints are mostly rigid. Therefore, before solving its motion model, the rigid body is briefly introduced. Describe the state of the rigid body and need to describe its position and attitude. The tandem robot is essentially a space kinematic chain consisting of the individual rods connected by the movement pair. To describe the relative position of each bar, usually using $\mathrm{D}-\mathrm{H}$ parameter method, that is, the introduction of connecting rod coordinate system. Just like Figure 2 [18].

\section{Coordinated Control of a Mobile Manipulator.}

The combination of mobile platforms and Manipulators first requires effective coordination of movement and operation. For some platforms, the direction of motion of the platform is limited by the nonholonomic constraints introduced by the pure rolling between the wheels and the ground. The combination of multi-link manipulator and platform results in the redundancy of the whole system. The solution of redundancy is the first problem encountered in coordination control [19]. At the same time, both mobile and Manipulator have complex dynamic models, and both There is a strong coupling between, in addition, the mobile platform is generally the quality of heavy, dynamic response is slow; and the Manipulator light weight, dynamic response fast. When people study each of the control problems, it is generally the first to consider the simplest case, as is the case with mobile Manipulators [20]. Wiens studied the effect of the motion of the platform on the manipulator performance of the manipulator by rotating the joint and the non-moving platform. The passive control was used to reduce the influence of the platform motion on the manipulator control. , But did not consider the control of the platform. It is assumed that the manipulator has been taught well and that the manipulator is in the preferred operating area and the optimal position is maintained through the control platform.

\section{Conclusions}

Mobile manipulator has made a lot of achievements in control theory and applied research, but its research is still unsatisfactory in some respects, so the existing problem is the research direction in this field. From the point of view of the controller design, the mobile manipulator has a very complicated dynamic model, so the design of the controller must take into account the complete dynamics model. Which is must include uncertainties such as environmental change, unknown external disturbances, obstacle avoidance, etc. otherwise the controller will not achieve the desired performance index. From the point of view of processing method, if the cost function method is adopted to solve the planning problem, different cost functions should be chosen according to different tasks, and the expected solution can be found by different optimization methods. From the control objectives: the design of the controller should give full play to the mobile manipulator movement and operation of the combination of advantages, which allows the manipulator and platform simultaneous movement to complete the task. In addition, many of the existing planning 
and control methods only through the computer simulation to verify the feasibility, but not in the actual mobile robot system to test, so the mobile robot control problems there are many to be done, especially in the country There is no real mobile robotic system

\section{References}

[1] Mirosław Galicki. Real-time Robotics and Autonomous Systems, 2016, No.23, P.13.

[2] Abdelfetah HENTOUT,Mohamed Ayoub MESSOUS,Brahim BOUZOUIA..IFAC Proceedings Vol (2014), p.47.

[3] M.H. Korayem,S.R. Nekoo. Robotics and Autonomous Systems, 2016 p.32.

[4] Y. Chen, F.Y. Xia. Journal of Chongqing University of Arts and Sciences, Vol. 35(2016), No.2, p.79. (In Chinese).

[5] Salima IFAC Proceedings Vol, 42(2009), No, 13.

[6] Grzegorz Pajak,Iwona Pajak. Archive of Mechanical Engineering, (2014), No. 61.

[7] Fu-Cai Liu,Li-Huan Liang,Juan-Juan Gao.International Journal of Automation \&amp; Computing,(2014),No.4,p.353.

[8]. T. Mouri, H. Kawasaki, K. Yoshikawa,et al:Chinese Journal of Mechanical Engineering,(2012),No.2,p.197.

[9] CHEN Kang,MA Chunxiang,ZHENG Maoqi,GAO Feng.Chinese Journal of Mechanical Engineering, Vol.2(2015),P.236.

[10]OTYC, ALBU-SCHAERA, KUGIA, et a1. IEEE Transactions on Robotics, Vol. 24(2008), No.2, p.416.

[11]S.W. Fan, Y.W. Liu, M.H. Jin, et al. Journal of Harbin Engineering University, Vol.30(2009), No.2, p.171.

[12]Jacob Wilson,Meaghan Charest,Rickey Dubay. Robotics and Computer Integrated Manufacturing, (2016), p.371.

[13]M. Benzaoui,H. Chekireb,M. Tadjine,A. Boulkroune.. Neurocomputing, 2016, p. 13.

[14]T. Mouri, H. Kawasaki, K. Yoshikawa,et al: Proceedings of the 2002 IEEE ICCA(2002), p.1288.

[15]P.F. Ji, Y.H. Liu, C.G. Sun. MECHANICAL ENGINEER,(2017), No.2, p.66.

[16]H. Liu, G. Hirziger. Journal of Xi An Jiao Tong University, Vol. 37(2003), No.4, p.331. (In Chinese).

[17]Amruta Mujumdar,Shailaja Kurode,Bhagyashri Tamhane. IFAC Proceedings Vol, 47(2014), No.1, p. 232.

[18]Xinxin Yang,Zhen Zhong. Dynamics and Terminal Sliding IFAC Proceedings Vol, 46(2013), No.20, p. 34.

[19]Daniela J. López-Araujo,Arturo Zavala-Río,Víctor Santibáñez,Fernando Reyes. IFAC Proceedings Vol, 45(2012), No.22, p.56.

[20]Lu Lu,Bin Yao,Zhibin Liu. FAC Proceedings Vol, 46(2013), No.5, p. 142. 